\title{
Serum thymidine kinase 1 is associated with Gleason score of patients with prostate carcinoma
}

\author{
SHUJING LI ${ }^{1 *}$, JIANPING ZHOU $^{2 *}$, YU WANG $^{3}$, KEQIN ZHANG $^{1 *}$, JUNJIE YANG $^{4}$, XINLING ZHANG $^{2}$, \\ CHUNMEI WANG ${ }^{2}$, HONGBO MA $^{5}$, JI ZHOU ${ }^{5}$, ELLEN HE ${ }^{5}$ and SVEN SKOG $^{5}$ \\ ${ }^{1}$ Department of Nephrology, Southwest Hospital, Third Military Medical University, Chongqing 400030; \\ ${ }^{2}$ Radioimmunity Center, Shaanxi Provincial People's Hospital, Xian, Shaanxi 710068; ${ }^{3}$ Health Management Center, \\ People's Liberation Army 180 Hospital, Quanzhou, Fujian 362000; ${ }^{4}$ Department of Urology, \\ Daping Hospital, Third Military Medical University, Chongqing 400042; ${ }^{5}$ Department of Medicine, \\ Sino-Swed Molecular Bio-Medicine Research Institute, Shenzhen, Guangdong 518057, P.R. China
}

Received November 14, 2017; Accepted July 3, 2018

DOI: $10.3892 / \mathrm{ol} .2018 .9345$

\begin{abstract}
The aim of the present was to assess whether serum thymidine kinase 1 (STK1) concentration is a useful biomarker for the screening of benign prostatic hyperplasia (BPH) or prostate malignancy. Serum samples were collected from 123 patients with prostate carcinoma prior to surgery, biopsy or androgen deprivation therapy and at 3,6 and 10 months following the procedure. A total of 205 patients with BPH and 266 healthy controls were also utilized. STK1 concentration and total prostate-specific antigen (PSA) were measured in patient serum by use of commercial assays. The pathological specimens (obtained from surgery or biopsy) were assessed according to Gleason scores (GS). STK1 concentration and total PSA were significantly higher in patients with prostate carcinoma compared with patients with BPH and healthy individuals. Furthermore, STK1 concentration was associated
\end{abstract}

Correspondence to: Professor Sven Skog, Department of Medicine, Sino-Swed Molecular Bio-Medicine Research Institute, Building A2/19f, Kexing Science Park, C. 1st Avenue, Gaoxin, Shenzhen, Guangdong 518057, P.R. China

E-mail: svenisak@icloud.com.

Dr Keqin Zhang, Department of Nephrology, Southwest Hospital, Third Military Medical University, 30 Gaotanyan, Shapingba, Chongqing 400030, P.R. China

E-mail: zhkn2000@sina.com

*Contributed equally

Abbreviations: TK1, thymidine kinase 1; STK1, serum thymidine kinase 1; STKa, serum thymidine kinase activity; PSA, prostate specific antigen; $\mathrm{PC}$, prostate cancer; $\mathrm{BPH}$, benign prostatic hyperplasia; GS, Gleason score; AUC, area under the curve; ROC, receiver operation characteristic; RP, radical prostatectomy; ISUP, International Society of Urological Pathology

Key words: prostatic carcinoma, benign prostatic hyperplasia, serum thymidine kinase 1, prostate-specific antigen, Gleason score with Gleason score, while total PSA was not. However, no association was identified between STK1 concentration and total serum PSA. A receiver operating characteristic analysis was performed on STK1 concentrations among patients with prostate carcinoma. The results demonstrated that the sensitivity and specificity were high, with an area under the curve (AUC) of 0.97. Although the sensitivity and specificity of total PSA were also high, the AUC value was relatively low (0.74). The results indicated that STK1 concentration is a more reliable prognostic biomarker than total PSA in respect to the GS system. Additionally, since STK1 concentration is associated with Gleason score, the use of biopsies to determine Gleason score may be replaced to some extent by the STK1 concentration test, thus reducing the discomfort of patients from which biopsies are obtained.

\section{Introduction}

Prostate cancer (PC) is the most commonly diagnosed non-skin cancer among men in Western countries, which carries a high mortality (1-4). However, trends have revealed a continuous decrease in the mortality of patients with prostate cancer $(2,4,5)$. This may be a result of novel treatments for PC. In China, the incidence rate of PC was low in $1960(0.48 / 100,000$ individuals), but increased by 2013 (13.33/100,000 individuals), due to a combination of factors, including ageing and dietary and lifestyle changes $(5,6)$.

The Gleason grading system was developed in the 1960s and was based on histopathological data obtained from radical prostatectomy (RP) (7); however, this system has remained unchanged for decades. The development of immunohistochemical staining has led to the identification of histological patterns that were misclassified in Gleason's original drawings, which has resulted in a clearer distinction between benign mimickers of PC (including adenosis and prostatic intraepithelial neoplasia) and PC itself (8). Due to the reclassification of the Gleason scoring system, in 2005, the International Society of Urological Pathology (ISUP) Consensus Conference disregarded the old numbers of the Gleason score (GS) of 5 or less as they were no longer 
considered to be representative of adenocarcinoma and as such had no prognostic value (9). GS 6 is now recommended as the lowest grade assigned to prostatic biopsy (10). In addition, numerous former GS 6 tumors have been reclassified as GS 7. Modern GS 6 tumors now exhibit a better prognosis than those described in older literature (11). Although GS is based on invasive and costly prostatic biopsies, it provides a definitive diagnosis and remains one of the most powerful prognostic predictors for patients with PC (12). This novel grading system was accepted by the World Health Organization for the 2016 edition of Pathology and Genetics: Tumors of the Urinary System and Male Genital Organs (11).

However, there are many limitations of the GS system that limit the effective determination of PC aggressiveness. These include tumor heterogeneity, biopsy-sampling error and variations in biopsy interpretation (13-15). The resulting uncertainty in risk assessment leads to a significant possibility of complication with associated pain $(13,14)$. Thus, the current evaluative methods of biopsy are often unable to stage individual patients accurately (15). Therefore, the GS system is much more complex than its original version, which may be problematic for clinicians and patients. However, we hypothesize that this may be overcome by combining the assessment of non-invasive biomarkers with GS, which may improve the prognostic/predictive testing system of PC.

The level of prostate-specific antigen (PSA) is the most important prognostic PC serum marker $(5,16)$. However, a discrepancy has been reported between needle biopsies and RP specimens $(6,17)$. GS value allocation depends on various clinical factors, including PSA cut-off values used (4 or $10 \mathrm{ng} / \mathrm{ml})$, number of prostate needle biopsies taken and experience level of the pathologist (18-20). The biopsy GS value is a primary factor for the selection of appropriate treatments, including RP, androgen deprivation therapy or conservative therapy (21-24). Together with clinical factors, pre-treatment PSA levels may improve the identification of patients who are at higher risk of mortality following surgery. These are primarily patients with a GS value of $\mathrm{G} 3+3$ following prostate biopsy, with a pre-operative PSA value of $\geq 10 \mathrm{ng} / \mathrm{ml}$ (18). There is different opinion about the PSA values of low-risk (4 ng/ml) or high-risk (10 ng/ml) patients with PC. However, there is no significant association between PSA expression and PC phenotype, and functional and tumorigenic heterogeneity (19). The use of PSA as a diagnostic marker is limited as it is also expressed in healthy prostate tissue and elevated circulating levels may be exhibited in patients with prostatitis, inflammation and BPH (20). In addition, PSA screening trials have demonstrated that many patients diagnosed with PC do not develop life-threatening disease (25-27). Clinical trials on PC screening demonstrated a limited benefit to patient survival (25-27). A meta-analysis of five randomized control trials indicated that PSA testing did not significantly decrease PC-specific mortality (25). Furthermore, PC can develop in patients whose PSA levels remain low (26). There was also no evidence of a reduction in PC mortality in the American Prostate, Lung, Colorectal and Ovarian Cancer Screening Trial (27). Given that opportunistic PSA screening practices in Canada are similar, it is unlikely that the introduction of a formal PSA screening program would reduce PC mortality (27). PSA is now commonly used in combination with RP or needle biopsy to distinguish benign from aggressive types of cancer, which may orient treatment application (10). Despite its poor specificity, PSA remains an integral component of statistical models that combine assorted patient specific variables (10).

Cancer is a disease of abnormal proliferating cells. Mutations in certain enzymes and proteins associated with cell growth regulation leads to uncontrolled proliferation and thus malignancy $(11,28)$. Therefore, the identification of proliferating biomarkers to improve the early detection of PC is important. Aside from PSA (29), serum thymidine kinase 1 (STK1) is a potential biomarker of proliferation that has been used to determine patient prognosis and treatment progress, either as STK activity (STKa) or STK1 concentration (STK1) $(5,30,31)$. STKa has become a more useful tool to assess patient prognosis, tumor treatment progress, relapse, follow-up and survival, particularly in solid tumors (5). However, there are few reports that determine STK1 application in PC. A previous study performed in 1996 was the first to assess STKa in relation to PC (32). In this study $(n=92)$, the mean value of STKa was compared with PSA and it was determined that there was a significant difference in mean STKa values between $\mathrm{PC}$, benign prostatic hyperplasia $(\mathrm{BPH})$ and healthy individuals (21). Li et al (33) demonstrated that STK1 was a reliable biomarker as it was able to discriminate between patients with PC, patients with BPH and healthy individuals [PC, $n=70$, $3.7 \pm 1.9 \mathrm{pM}$, sensitivity 0.72 at a cut-off of $2.0 \mathrm{pM}$; $\mathrm{BPH}, \mathrm{n}=40$, $1.3 \pm 0.4 \mathrm{pM}$; healthy individuals, $\mathrm{n}=40,0.8 \pm 0.3 \mathrm{pM}]$. STK1 concentrations above the level of $2.0 \mathrm{pM}$ was also associated with clinical stage $\left[\mathrm{T}_{1} 62.9 \%(22 / 35), \mathrm{T}_{2} 73.7 \%(14 / 19), \mathrm{T}_{3} 80.0 \%\right.$ $(8 / 10)$ and $\left.\mathrm{T}_{4} 100.0 \%(6 / 6)\right]$. Jagarlamudi et al (34) compared the STKa and concentration of STK1 in patients $(n=47)$ with PC to healthy blood donors. The results demonstrated that STKa and STK1 concentration differed significantly between patients with PC and healthy individuals (35). Furthermore, the STKa of patients with well-differentiated $(\mathrm{GS} 5+6) \mathrm{PC}$ tumors were at similar levels to healthy individuals, while the corresponding values in patients with moderately/poorly differentiated (GS7+8) tumors were significantly elevated (35). However, the STK1 concentration in patients with well- and moderately/poorly-differentiated tumors was significantly higher compared with healthy individuals (35). Additionally, while STKa and STK1 concentrations were similar (0.96), a difference in sensitivity (STKa 0.15; STK1 concentration 0.64) and AUC-value (STKa 0.69; STK1 concentration 0.88) was identified (35). Thus, STK1 concentration may serve as a prognostic biomarker for the early detection (GS5+6) of PC. Additionally, TK1 immunohistochemical staining demonstrated that TK1 expression in PC tissues was associated with time of recurrence and development of metastasis, indicating that it may also serve as a useful biomarker for patients with PC (35) [n=103, GS 6, mouse TK1 IgG clone 5; SSTK, Ltd., Shenzhen, China).

The present study extended the previous research into STK1 concentration in patients with PC by comparing STK1 concentration with total PSA in serum and assessing Gleason score values of prostate tumor tissue. The aim of the current study was to further explore the possibility that STK1 concentration could be used as a prognostic biomarker in patients with BPH and PC. 


\section{Materials and methods}

Patients. All procedures performed in the present study involving human participants were in accordance with the ethical standards of the institutional and/or national research committee and with the 1984 Declaration of Helsinki with its later amendments or comparable ethical standards. The present study was also approved by the Ethics Committee of the Health Management Centre of People's Liberation Army (PLA) 180 Hospital (Quanzhou, China; approval no. LL2009003). Informed consent was obtained from all participants of the present study.

The following criteria of the patients were assessed: Age, Gleason score, TK1 levels and PSA levels, plus the 5-year follow-up of 1 patient, including detailed medical data. The following criteria were excluded: ID, phone number, home address, data when visiting the hospital, routine blood and urine test results, ultrasound and advanced imaging results, and their objective response to treatment.

Based on the Gleason scoring system of core needle biopsies, as described by the ISUP guideline revision in 2005, the diagnosis of the glandular dedifferentiation level was performed. Certain patients $(n=26)$ refused to undergo biopsies and thus the Gleason score could not be determined. Next, the patients underwent surgery by laparoscopic radical prostatectomy (RP) and further routine treatment was provided individually, according to previously described guidelines (36), at Daping Hospital, Third Military Medical University (Chongqing, China) and at Shaanxi Provincial People's Hospital (Xian, Shaanxi, China).

The effect of the treatment was evaluated by the changes in the STK1 values. Since this is a study based on routine clinical data, only 18 patients were available for monitoring of the effect of the treatment.

Survival assessment was performed in 51 patients only (51/123), due to limited access to the patients once they left the hospitals. The patients or their families were contacted by phone every year to determine if they remained alive. One of the patients succumbed during the follow-up period of 5 years. The clinical data of this patient during the 5-year follow-up are provided in the Results section. In the first treatment cycle that started in May 2010, the patient was treated with $250 \mathrm{mg}$ flutamide orally 3 times per day and with $0.1 \mathrm{mg}$ diphereline intramuscular once per month, with each treatment lasting for 3 years. In the second treatment cycle that started in May 2013, the patient was first treated by one infusion dose of $4 \mathrm{mg}$ zoledronic acid to treat possible metastasis, followed by $50 \mathrm{mg}$ bicalutamide once orally and then $250 \mathrm{mg}$ flutamide 3 times per day until the patient succumbed in February 2016. The patients were also investigated by ultrasound B (DC-6E; Shenzhen Mindray Bio-Medical Electronics Co., Ltd. China) and computed tomography (CT) (Brilliance $1 \mathrm{CT}$; Philips Medical Systems B.V., Eindhoven, The Netherlands).

A summary of patient age and pathology/Gleason score are presented in Table I. A total of 123 patients with PC pre-operative were investigated between March 2008 and December 2009. Tissue specimens and serum samples were collected from 108 men (mean age, 72.9 \pm 9.6 years; age range, 34-93 years) with prostate adenocarcinoma who had not yet received surgery at Daping Hospital and Research Institute of
Table I. Gleason score, number and age of participants involved in the present study. ${ }^{\mathrm{a}}$

\begin{tabular}{lcc}
\hline Gleason score & Number of patients & Age range (years) \\
\hline G2+G3 & 7 & $64-78$ \\
G4+G5 & 15 & $66-93$ \\
G6 & 10 & $56-81$ \\
G7 & 49 & $34-85$ \\
G8+G9 & 16 & $66-80$ \\
Not available & 26 & $62-78$ \\
\hline
\end{tabular}

${ }^{\mathrm{a} A l l}$ patients were diagnosed with prostate adenocarcinoma.

Surgery of the Third Military Medical University (Chongqing China) between March and December 2008. Tissue specimens and serum samples were also collected from 15 men (mean age, 73.5 \pm 8.1 years; age range, 54-84 years) with prostate adenocarcinoma who had not received surgery at Shaanxi Provincial People's Hospital (Xian, China) between January and December 2009.

Pre-operative tissue and serum samples were collected from patients with BPH tumors ( $\mathrm{n}=205$; mean age, $71.8 \pm 7.9$ years; age range, 57-91 years) at Shaanxi Provincial People's Hospital (Xian, China) between January and December 2009. Serum samples from healthy individuals $(n=266$; mean age, $60.6 \pm 7.8$ years; age range, 51-87 years) who had no evidence of contagious or cancerous disease were also collected at Shaanxi Provincial People's Hospital (Xian, China) and at the Health Management Centre of PLA 180 Hospital (Quanzhou, China) between January and December 2009.

The operative and biopsy specimens were processed according to the Gleason system. Two experienced genitourinary pathologists assessed and allocated primary and secondary GS. The GS numbers utilized in the present study were the sum of the primary and secondary scores (for example: GS2+GS3=GS5). Patients were divided into five groups: GS2-3, GS4-5, GS6, GS7 and GS8-9. Discrepancies were resolved by a joint review of the slides. Of the patients included in the present study, $65 \%$ exhibited a Gleason score of GS7 or higher and were considered to be high risk.

The tissues of the biopsies were fixed prior to the examination. The specimens $(5-\mu \mathrm{m}$ thick) were fixed with neutral formaldehyde (4\%) for $>2 \mathrm{~h}$, followed by dehydration in $70 \%$ ethanol (10 $\mathrm{min}), 80 \%$ ethanol $(20 \mathrm{~min}), 95 \%$ ethanol (30 min), anhydrous ethanol (30 min), xylene (I) $(5 \mathrm{~min})$, xylene (II) (5 min) and xylene (III) (5 min). The specimens were paraffin-embedded at $62^{\circ} \mathrm{C}(8$ mins each, 3 times). Prior to examination, the tissues were deparaffinized and examined at room temperature.

It is important to note that STK1 and PSA were not determined in the same group of patients due to the routine clinical nature, and thus the number of STK1 and PSA patients investigated was not equal.

TK1 assay. STK1 concentrations were assessed using a commercial kit (Thymidine Kinase 1 Cell Cycle assay kit; cat. no. 24/48T) based on an enhanced chemiluminescent 
dot blot assay as described by the manufacturer (Sino-Swed Tongkang Bio-Tech Inc., Shenzhen, China). The collection of serum was performed after patients had fasted for 12-14 h (7.30 am-10.00 am) and samples were analyzed within $3 \mathrm{~h}$ of whole blood centrifugation $\left(400 \mathrm{x} \mathrm{g}, 22-25^{\circ} \mathrm{C}, 8-10 \mathrm{~min}\right)$. If not analyzed immediately, samples were stored at $-20^{\circ} \mathrm{C}$ for a maximum of 4 weeks. Although the serum samples were analyzed within 4 weeks, the serum may be stored for $\geq 1$ year at $-20^{\circ} \mathrm{C}$ and still maintain TK1 in good condition. Samples comprising $3 \mu \mathrm{l}$ serum were directly applied to nitrocellulose membranes in duplicate. Serum samples were then probed with chicken anti-human TK1 IgY polyclonal antibody (dilution 1:500; Thymidine Kinase 1 Cell Cycle assay kit, cat. no. 24/48T; Sino-Swed Tongkang Bio-Tech Inc.) raised against a peptide (residue 195-225 of human TK1, amino acid sequence: GQPAG PDNKE NCPVP GKPGE AVAAR KLFAPQ; Multiple Peptide Systems, San Diego, CA, USA). The TK1 peptide was dotted onto membranes at different concentrations $(2.2,6.6$ and $20 \mathrm{pM})$ as an extrapolated standard. The intensity of spots on the membrane was determined using a CIS-1 Imaging System (Sino-Swed Tongkang Bio-Tech Inc.). From the intensities of the TK1 standard of known concentrations, the STK1 concentration value was calculated and expressed as pM. The present study selected a STK1 concentration cut-off value of $1.0 \mathrm{pM}$, as it exhibited a higher sensitivity compared with a concentration of $2.0 \mathrm{pM}$ (0.84 vs. 0.61). All hospitals utilized the same TK1 assay kit (cat. no. 24/48T Sino-Swed Tongkang Bio-Tech Inc.) in accordance with the manufacturer's protocol when determining the TK1 level from serum samples of the membrane.

PSA assay. PSA levels were determined using an electrochemiluminescence immunoassay. The electrochemiluminescence automatic immunoassay analyzer functions using paramagnetic particles as a solid phase, a biotin-streptavidin-detection system and two-dimensional bar code technology. It is a highly sensitive light detection system that provides excellent low-end sensitivity and a broad dynamic measuring range. The reference cut-off value in the present study was $4.0 \mathrm{ng} / \mathrm{ml}$, which was recommended by the PSA-kit supplier. The PSA values were considered positive or negative when PSA levels were above or below the cut-off value, respectively. The three hospitals involved in this study utilized two different automatic machines for PSA determination [i) Beckman Coulter UniCel ${ }^{\circledR}$ DxI 800; Chongqing Huanuo Medical Biotechnology Co., Ltd., Chonqing, China; PSA kit, cat. no. 37200; and Nanchang Qicheng Pharmaceutical, PSA kit, cat. no. 37200; ii) Chemiluminescence Immunoanalyzer, Liason Type 2229; Beijing Zhong Yi Kai Chuang Co., Ltd., Beijing, China; PSA kit, cat. no. 314381]. Each machine produced PSA values in the same range: Low $(1 \pm 1 \mathrm{ng} / \mathrm{ml})$, medium $(12 \pm 14 \mathrm{ng} / \mathrm{ml})$ and high $(41 \pm 51 \mathrm{ng} / \mathrm{ml})$ values corresponded to healthy individuals, patients with $\mathrm{BPH}$ and patients with PC, respectively.

Statistical analysis. Data are presented as the mean \pm standard deviation. For the comparison of STK1 concentration and PSA levels among the different groups of patients investigated, one-way analysis of variance followed by a post-hoc least significant difference test was performed. SPSS version 19 was utilized for statistical analysis (IBM Corp., Armonk, NY,
USA). Regression analysis was performed using Microsoft Excel (version 15.37; Microsoft Corporation, Redmond, WA, USA). Receiver operating characteristic (ROC) analysis was performed using the ROC program within the Analyse-It statistical program version 2.2 (Analyse-It Software, Ltd., Leeds, UK). The P-values presented in ROC analysis were calculated using the ROC program. $\mathrm{P} \leq 0.05$ was considered to indicate a statistically significant result.

\section{Results}

STK1 concentration and total PSA of healthy, BPH and malignant men. The mean values of STK1 concentration and total PSA in patients with BPH and PC were significantly higher compared with healthy individuals. The mean STK1 concentrations and total PSA in patients with PC was also significantly higher compared with patients with BPH (Tables II and III; Fig. 1). Furthermore, 4\% of patients with PC could be distinguished from healthy individuals and those with BPH using STK1 concentrations. The corresponding value based on total PSA was $28 \%$ (data not shown).

STK1 concentration, total PSA and Gleason score. STK1 concentration was associated with GS value; however, the association of total PSA with GS value was unclear (Tables IV and V, Fig. 2). Of patients with PC, $17.5 \%$ of those staged at GS7-GS9 exhibited STK1 concentrations $>3.5 \mathrm{pM}$ and $10.3 \%<1.0 \mathrm{pM}$ (Fig. 2A). Furthermore, $14.3 \%$ of patients staged at GS7-GS9 exhibited PSA values above the cut-off value $(4.0 \mathrm{ng} / \mathrm{ml})$. However, there was no significant difference in PSA value among patients with low GS scores (Table V and Fig. 2B). Thus, STK1 concentration is able to identify patients with high (GS7-GS9) and low (GS3-GS4) GS scores, while PSA only identifies patients with a high GS score (G7-G9).

Association between STK1 concentration and total PSA in serum. No significant association between STK1 concentration and total PSA in the serum of patients with BPH or PC was identified (Fig. 3).

Monitoring and survival. Of the 123 patients with PC, 18 cases were monitored for up to 10 months following prostate biopsy and radical prostectomy (Table VI). STK1 concentration decreased significantly at 3,6 and 10 months compared with the STK1 values prior to surgery. No PSA values were obtained.

Of the 123 men with prostate malignancy, 51 were followed up for 5 years. A total of $98 \%(50 / 51)$ of patients survived following these 5 years.

To confirm that STK1 and PSA values were associated with the effect of treatment, the data of one individual are presented. The individual that succumbed was found to have stage II BPH at the age of 85 and exhibited frequent urination, with an STK1 concentration of $1.5 \mathrm{pM}$ and a PSA value of $140 \mathrm{ng} / \mathrm{ml}$. The ultrasound (Philips iU22) scan exhibited calcification and a retention cyst $(5.8 \times 4.8 \times 5.8 \mathrm{~cm})$. Furthermore, $\mathrm{CT}$ results revealed prostate hyperplasia. Following 3 months, $\mathrm{BPH}$ progressed into PC. A biopsy was performed and a GS of GS4+3 was determined. STK1 concentration was increased to 
Table II. STK1p (pM) mean value \pm standard deviation of healthy individuals and patients with benign prostatic hyperplasia or prostate malignancy.

\begin{tabular}{lccc}
\hline Type & Healthy & Benign/hyperplasia & Malignant \\
\hline Mean & $0.4^{\mathrm{a}, \mathrm{b}}$ & $1.3^{\mathrm{b}}$ & 2.5 \\
Standard deviation & 0.3 & 0.7 & 2.0 \\
Count & 356 & 205 & 123 \\
Max & 2.1 & 5.1 & 14.7 \\
Min & 0.0 & 0.1 & 0.2 \\
\hline
\end{tabular}

${ }^{\mathrm{a}} \mathrm{P}<0.001$ vs. benign/hyperplasia; ${ }^{\mathrm{b}} \mathrm{P}<0.001$ vs. malignant. STK1, serum thymidine kinase 1; Max, maximum; Min, minimum.

Table III. PSA (ng/ml) mean value \pm standard deviation of healthy individuals and patients with benign prostatic hyperplasia or prostate malignancy.

\begin{tabular}{lccc}
\hline Type & Healthy & Benign/hyperplasia & Malignant \\
\hline Mean & $1.3^{\mathrm{a}, \mathrm{b}}$ & $11.8^{\mathrm{c}}$ & 41.2 \\
Standard deviation & 0.9 & 14.1 & 51.3 \\
Count & 76 & 56 & 97 \\
Max & 4.0 & 66.6 & 153.0 \\
Min & 0.2 & 0.5 & 0.1
\end{tabular}

${ }^{\mathrm{a}} \mathrm{P}<0.001$ vs. benign/hyperplasia; ${ }^{\mathrm{b}} \mathrm{P}<0.01$ vs. malignant; ${ }^{\mathrm{c}} \mathrm{P}=0.021$ vs. malignant. PSA, prostate-specific antigen; Max, maximum; Min, minimum.

4.5 pM. The individual was treated with successful intermittent androgen therapy (flutamide + diphereline). Following 3 months, STK1 was decreased to $2.6 \mathrm{pM}$ and following a further 3 months, STK1 concentration reached a value of $0.6 \mathrm{pM}$, which is a result that corresponds to that of healthy individuals. PSA was also decreased to normal values ( $<4 \mathrm{ng} / \mathrm{ml}$ ). However, 48 months following the start of treatment, the PSA value increased to $5.5 \mathrm{ng} / \mathrm{ml}$. As a result, the individual received an additional cycle of treatment (bicalutamide + flutamide + diphereline) and following a further 12 months, PSA continued to increase $(11.75 \mathrm{ng} / \mathrm{ml})$. STK1 concentration was not assessed during the treatment period. A total of 62 months after the start of treatment, the individual succumbed at the age of 91 as a result of type III hypertension, chronic bronchitis, diabetes, coronary heart disease, fatty liver and multiple cerebral infarctions (data not shown)

ROC analysis of STK1 and total PSA. To investigate the use of STK1 concentration and PSA in the screening of PC, an ROC statistical analysis was performed, the results of which are presented in Table VII and Fig. 4. For patients with PC, the AUC value of STK1 was 0.97. At a cut-off concentration of $1.00 \mathrm{pM}$, the sensitivity and specificity of STK1 concentration were at 0.84 and 0.96 , respectively, and exhibited a likelihood of (+) 124.6 (data not shown). At a cut-off concentration of
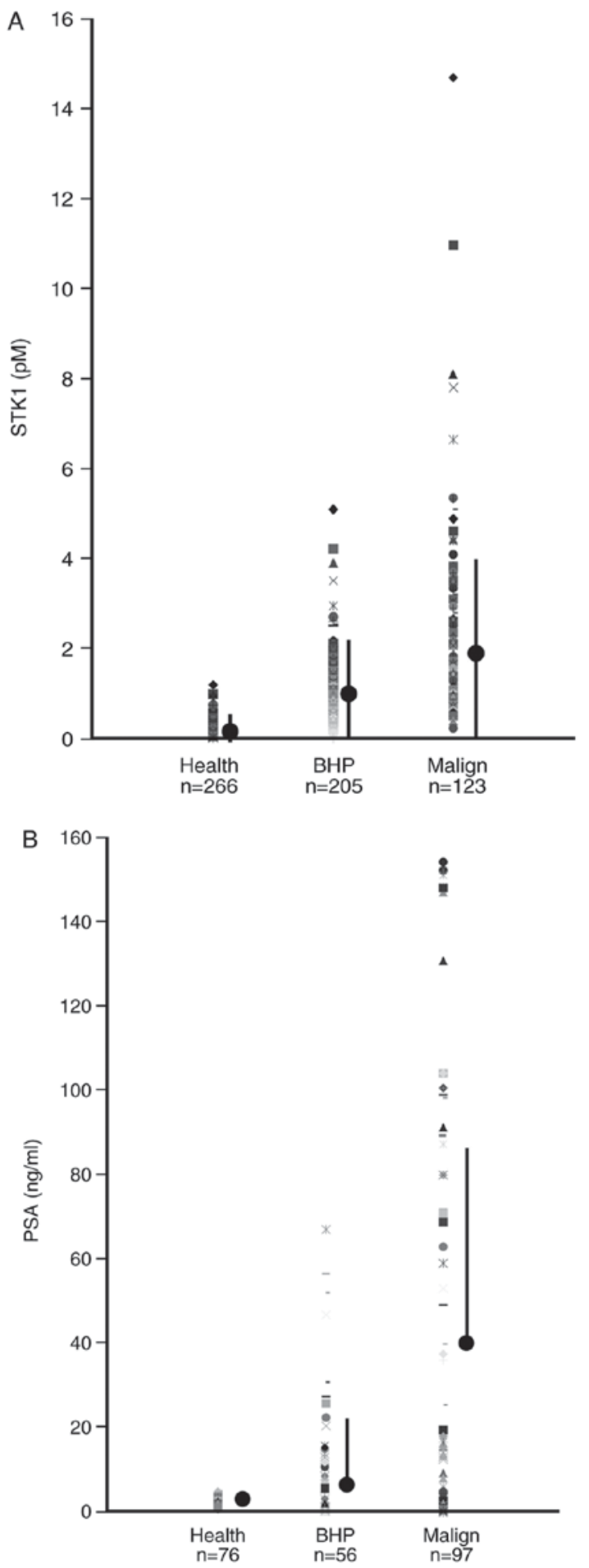

Figure 1. (A) STK1 concentration and (B) PSA values of healthy individuals and patients with BPH or prostate carcinoma. STK1, serum thymidine kinase 1; PSA, prostate specific antigen; Health, healthy individuals; BPH, benign prostatic hyperplasia; Malign, prostate carcinoma.

$2.00 \mathrm{pM}$, the sensitivity and specificity values of STK1 were 0.60 and 0.99 , respectively and exhibited a likelihood value of (+) 214.26. Similar values were obtained for patients with BPH tumors (Table VII). This indicates that the concentration of STK1 may be used in the screening of patients with BPH or PC. The sensitivity and specificity of total PSA in patients with PC were 0.62 and 0.93 , respectively. Furthermore, the likelihood value was (+) 9.40 and the AUC value was 0.74 (Table VII). 
Table IV. STK1p (pM) values in relation to Gleason score.

\begin{tabular}{lccccc}
\hline Type & G2+3 & G4+5 & G6 & G7 & G8+9 \\
\hline Mean & $1.0^{\mathrm{a}, \mathrm{b}}$ & $1.5^{\mathrm{a}, \mathrm{b}}$ & 2.1 & 3.0 & 2.9 \\
Std & 0.5 & 0.8 & 1.3 & 1.9 & 2.9 \\
Count & 7 & 16 & 10 & 49 & 16 \\
Max & 1.8 & 3.2 & 4.4 & 10.8 & 8.1 \\
Min & 0.4 & 0.4 & 0.5 & 0.8 & 1.0 \\
\hline
\end{tabular}

${ }^{\mathrm{a}} \mathrm{P}<0.01$ vs. $\mathrm{G} 7$; ${ }^{\mathrm{b}} \mathrm{P}<0.05$ vs. G8+9. STK1, serum thymidine kinase 1 ; Max, maximum; Min, minimum.

Table V. PSA (ng/ml) values in relation to Gleason score.

\begin{tabular}{lccccc}
\hline Type & G2+G3 & G4+G5 & G6 & G7 & G8+G9 \\
\hline Mean & 42.8 & $18.6^{\mathrm{a}, \mathrm{b}}$ & $11.5^{\mathrm{c}}$ & 47.5 & 72.0 \\
Std & 44.5 & 28.8 & 39.1 & 53.5 & 63.1 \\
Count & 4 & 18 & 8 & 48 & 9 \\
Max & 98.2 & 100.0 & 79.6 & 153.0 & 151.0 \\
Min & 1.5 & 0.1 & 0.1 & 0.1 & 1.8 \\
\hline
\end{tabular}

${ }^{\mathrm{a} P}<0.05$ vs. G7; ${ }^{\mathrm{b}} \mathrm{P}<0.01$ vs. G8+G9; ${ }^{\mathrm{C}} \mathrm{P}<0.05$ vs. G8+G0.PSA, prostate specific antigen; Max, maximum; Min, minimum.

Table VI. Mean \pm standard deviation of STK1 (pM) prior to and 3, 6 and 10 months following radical prostatectomy.

\begin{tabular}{lcccc}
\hline & & \multicolumn{3}{c}{ Months } \\
\cline { 3 - 5 } Type & Prior to procedure & 3 & 6 & 10 \\
\hline Mean & 2.9 & $1.4^{\mathrm{a}}$ & $1.0^{\mathrm{a}}$ & $1.0^{\mathrm{a}}$ \\
Std & 1.8 & 0.9 & 0.4 & 0.4 \\
Count & 12 & 16 & 9 & 4 \\
Max & 6.3 & 3.7 & 1.8 & 1.5 \\
Min & 0.9 & 0.4 & 0.5 & 0.5 \\
\hline
\end{tabular}

Type ${ }^{\mathrm{P}}<0.01$ vs. value prior to procedure. The lower patient numbers prior to radical prostatectomy $(n=12)$ compared with those following radical prostatectomy is due to certain patients not establishing STK1p prior to radical prostatectomy. STK1, serum thymidine kinase 1; Max, maximum; Min, minimum.

Similar values were identified in patients with BPH. Although the specificity and likelihood values were high, the AUC was relatively low (0.74) in patients with $\mathrm{PC}$, indicating that total PSA is a less reliable method of prostate screening compared with STK1.

\section{Discussion}

Although the Gleason grading system has undergone significant revisions, it still has problems that may potentially impact
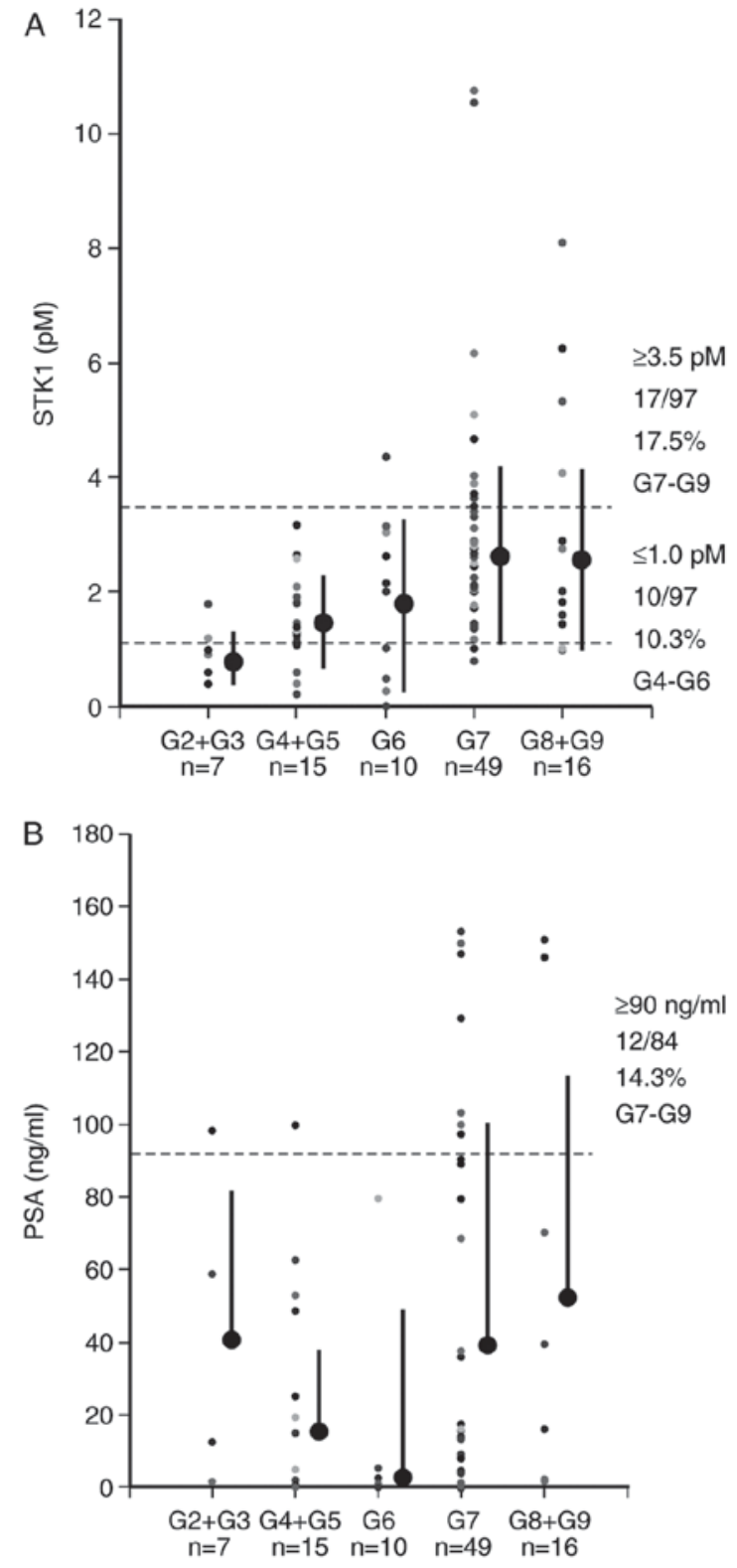

Figure 2. (A) STK1 concentration and (B) PSA values in association with the Gleason score of patients with prostate carcinoma. STK1, serum thymidine kinase 1; PSA, prostate specific antigen; G, Gleason score. The dotted lines in (A) indicate patients with STK1 values $\geq 3.5$ or $\leq 1.0 \mathrm{pM}$. The dotted line in (B) indicate patients with PSA values $\geq 90 \mathrm{ng} / \mathrm{ml}$.

patient care. Herein the current state of PC grading, with focus on the current guidelines for the Gleason grading system and recent changes from the 2014 ISUP Consensus Conference on Gleason Grading of Prostatic Carcinoma (11) will be discussed.

It is important to note that the present study was not a clinical trial following specific criteria, but was based on data collected during routine clinical work. This may limit the reliability of the conclusions drawn. However, the current study may indicate if STK1 and/or PSA can be used for the diagnosis of PC. The current study was also limited by the number of cases included ( $\mathrm{PC}, \mathrm{n}=123$; $\mathrm{BPH}, \mathrm{n}=205$; healthy controls, n=266). Further studies are thus required to confirm the conclusions drawn.

TK1 expression in tumor tissues and STK1 concentration in patients with $\mathrm{PC}$ have been demonstrated to be reliable 
Table VII. ROC analysis of STK1p and PSA.

\begin{tabular}{|c|c|c|c|c|c|}
\hline Type & Sensitivity & Specificity & Likelihood(+) & AUC & P-value \\
\hline \multicolumn{6}{|l|}{ STK1, cut-off 2.0 pM } \\
\hline Healthy vs. malignant & 0.60 & 0.99 & 124.61 & 0.97 & $<0.0001$ \\
\hline Healthy vs. benign & 0.09 & 0.99 & 30.85 & 0.89 & $<0.0001$ \\
\hline Benign vs. malignant & 0.65 & 0.95 & 11.10 & 0.74 & $<0.0001$ \\
\hline \multicolumn{6}{|l|}{ PSA, cut-off $4.0 \mathrm{ng} / \mathrm{ml}$} \\
\hline Healthy vs. malignant & 0.62 & 0.93 & 9.40 & 0.74 & $<0.0001$ \\
\hline Healthy vs. benign & 0.71 & 0.99 & 54.30 & 0.92 & $<0.0001$ \\
\hline Benign vs. malignant & 0.62 & 0.29 & 0.87 & 0.54 & 0.18 \\
\hline
\end{tabular}

P-values were estimated using the ROC statistical program (Analyse-It statistical program version 2.2). STK1, serum thymidine kinase 1; PSA, prostate specific antigen; AUC, area under the curve; ROC, receiver operating characteristic.
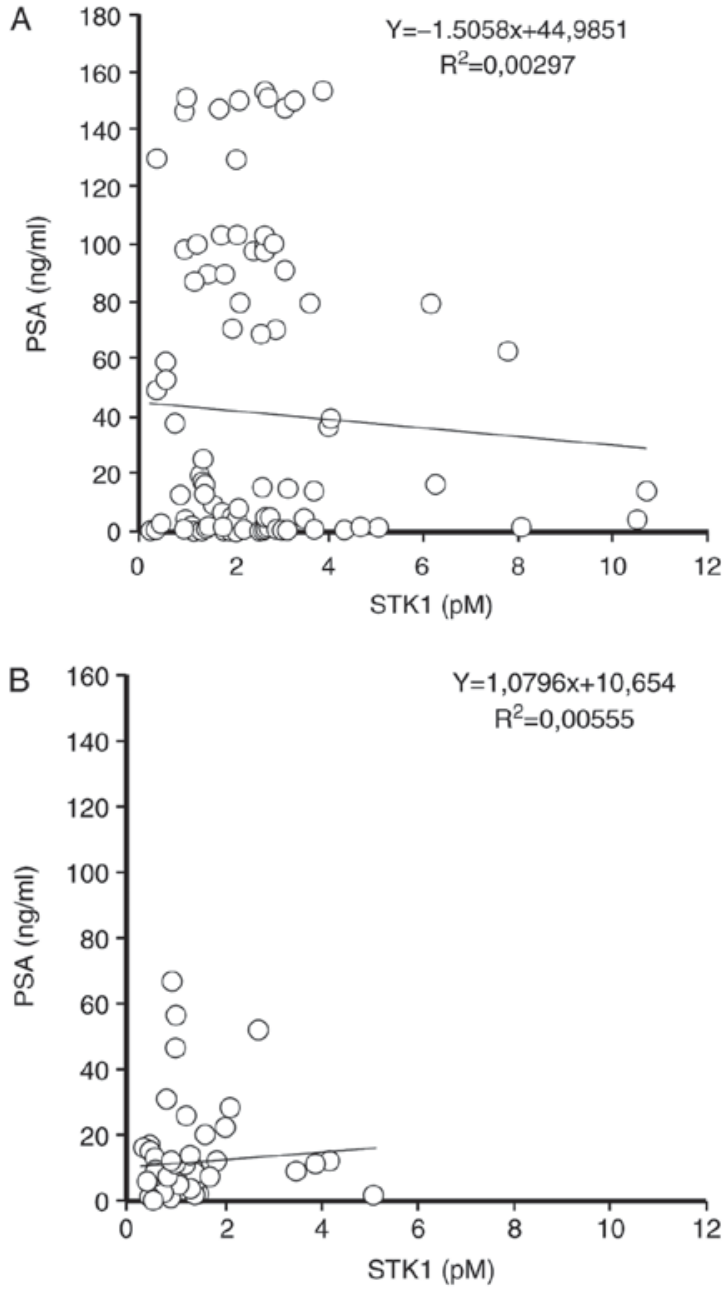

Figure 3. STK1 concentration vs. PSA in serum of (A) patients with prostate carcinoma and (B) patients with benign prostate hyperplasia. STK1, serum thymidine kinase 1; PSA, prostate specific antigen.

biomarkers for tumor recurrence and patient survival, as well as for cancer treatment monitoring for complete remission, partial remission, stable disease and progressive disease, and in leukemia, lymphoma and solid tumors of different types $(28,31)$. TK1 is a kinase enzyme involved
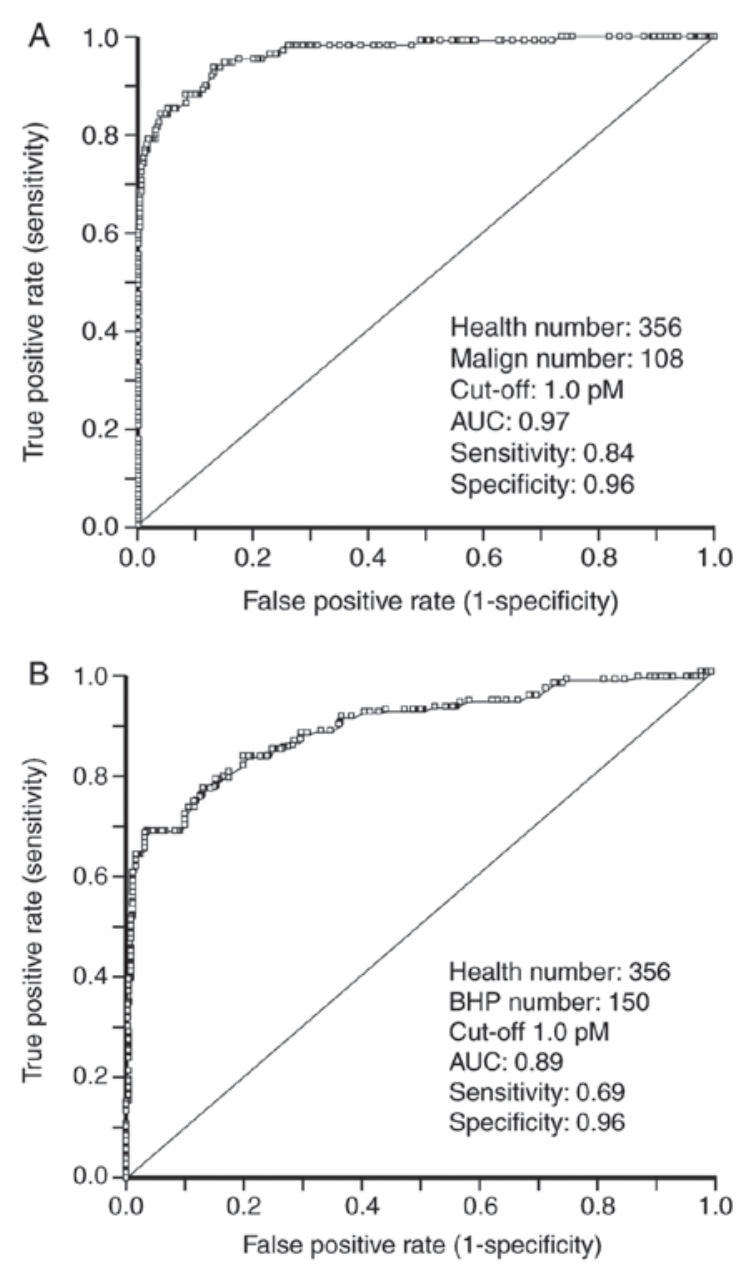

Figure 4. Receiver operating characteristic plot of (A) patients with prostate carcinoma and (B) patients with BPH compared with healthy individuals. Health, healthy individuals; Malign, prostate carcinoma; AUC, area under the curve; $\mathrm{BPH}$, benign prostatic hyperplasia.

in the synthesis of DNA and is a part of the thymidine salvage pathway, functioning to phosphorylate thymidine to thymidine monophosphate $(28,31)$. STK1 was originally measured by its activity (STKa). However, the STKa test was primarily used for patients with leukemia and lymphoma, as 
its use in solid tumors was limited. However, the updated STKa assay is now used in certain solid tumors, including breast and lung carcinoma. The development of chicken immunoglobulin (Ig) Y anti-TK1 antibodies made it possible to determine TK1 expression levels in various solid human tumors and TK1 concentrations in patient serum. In addition, IgY anti-TK1 antibodies exhibit no cross-reactivity in human serum and thus could be used in the screening of healthy individuals. IgG anti-TK1 antibodies currently on the market exhibit high levels of un-specific binding in human serum when utilized for the screening of healthy individuals. Thus far, we have investigated 20 different types of solid tumor using IgY anti-TK1 antibodies with convincing results (31) Using the IgY anti-TK1 antibody, TK1 expression in prostate tumor tissue determined by immunohistochemistry was demonstrated to be associated with relapse following RP (35).

The results of the present study were consistent with those from Jagarlamudi et al (34), who demonstrated that STK1 correlated to Gleason score. The study utilized the TK1 IgY antibody, which indicated that they were reliable. The close correlation between STK1 concentration and GS scores may potentially reduce the use of biopsy when screening for PC risk. The present study demonstrated that $10 \%$ of patients with low Gleason scores (GS3-GS4) and $17 \%$ of patients with high Gleason scores (GS7-GS9) could be identified via STK1 concentrations. Biopsies of any tissue, particularly that of the prostate, is unpleasant for patients and the reduction of its use would be advantageous. In a previous study based on the clinical trial Stockholm3 model (37) using a cohort of 145,905 individuals, a combination of PSA, clinical variables, established biomarkers and novel plasma protein biomarkers were utilized. The aim of this study was to determine a method by which to reduce the number of biopsies, since a biopsy is a painful process for a patient to endure. It was determined that the number of biopsies among individuals with a high risk of prostate carcinoma (GS7 or higher) was reduced by $32 \%$ and reduced by $44 \%$ among men with BPH (37). This is a promising improvement and may avoid unpleasant biopsies in men at risk of PC. To further improve these results, STK1 concentration could be utilized as an additional serum biomarker in the STHLM3 model.

The present study identified that no healthy men exhibited high total PSA values; however, a number of patients with BPH and PC exhibited low and high total PSA values, respectively, making it of limited use in prostate screening. The results also revealed that $28 \%$ of patients with PC exhibited higher total PSA values compared with those with BPH. Thus, although total PSA did not correlate to Gleason score, the individual value of total PSA may be used to identify certain individuals with a high risk of PC. Since the corresponding value of STK1 concentration was 4\%, it appears that total PSA is more reliable. However, it should be noted that STK1 concentration is associated with the growth rate of tumors, which is an important prognostic factor, while PSA is not (20). This indicates that the $4 \%$ of patients with PC identified by STK1 concentration possess a high risk, while the $28 \%$ identified by total PSA exhibit prostate tumors with a low proliferation rate and thus a better prognosis. Therefore, STK1 concentration may be a more reliable biomarker than total PSA. However, the present study recommends that PSA (total PSA or free-PSA) and STK1 concentration should be used in combination as they represent different properties of prostate tumors that are important for the assessment of risk.

In the current study, STK1 concentration was compared with total PSA in serum. However, the results did not identify any association between these two biomarkers. Furthermore, unlike STK1, total PSA was not associated with GS. However, certain cases exhibited high total PSA (>120 ng/ml) with a GS of G7-G9. These results confirm that total PSA has a limited use, as aforementioned. However, it should be noted that total PSA was measured in the present study, which meant that free-PSA was not excluded and thus may have produced different results. In a health screening of 486,085 people conducted at the 180 PLA Hospital (Quanzhou, China), 12,530 men were assessed for STK1 concentration and free-PSA. It was determined that no correlation existed between STK1 and free-PSA (unpublished data).

Although STK1 concentration was associated with GS, the question still remains as to whether STK1 concentration is sensitive and specific enough to be utilized in the screening of PC risk. To address this, the current study performed ROC statistical analysis. The sensitivity, specificity and likelihood (+) of STK1 were high and the AUC value was 0.97, which indicated that the determination of STK1 concentration may be a useful test for the screening of individuals for PC risk. The corresponding AUC value of PSA was 0.74. Although the ROC analysis of STK1 concentration presented a high AUC value, with a significantly higher mean value in patients with STK1 compared with patients with $\mathrm{BPH}$, only $4 \%$ of individuals with PC exhibited an STK1 concentration value above those with BPH. This limits the use of the STK1 assay when attempting to distinguish those with $\mathrm{BPH}$ from those with PC. However, STK1 concentrations were significantly higher in patients with PC compared with healthy individuals, in mean and individual values. In addition, in a previous health screening meta-analysis on 35,365 patients, it was demonstrated that those with moderate/severe hyperplasia, including prostate BPH, who exhibited high STK1 concentrations, had a 3-5 times higher risk of progression to malignancy (38). Thus, although there is a limited possibility to distinguish between patients with BPH and patients with PC, those diagnosed with BPH that exhibit high STK1 concentrations should be associated with a high risk of PC development later in life.

In conclusion, the results indicated that STK1 concentrations are significantly higher in patients with BPH and PC compared with healthy individuals, indicating that the determination of STK1 concentration may be used for the screening of prostate complications. However, due to the overlapping of individual STK1 values between patients with BPH and those with PC, distinguishing between these two groups via the assessment of STK1 concentration alone may be inefficient. It is likely that patients with BPH that exhibit high STK1 concentrations have an increased risk of progression to malignancy. Since STK1 concentration is associated with GS, it may be possible to reduce the number of biopsies obtained from men with suspected prostate $\mathrm{BPH} /$ malignancy by determining STK1 concentration. 


\section{Acknowledgements}

This study was supported by the Southwest Hospital, Third Military Medical University (Chongqing, China), the Shaanxi Provincial People's Hospital, the PLA 180 Hospital and Daping Hospital, Third Military Medical University, which provided facilities for running the tests, and by Sino-Swed Molecular Bio-Medicine Research Institute for development of the TK1 IgY antibody.

\section{Funding}

This study was supported by the Southwest Hospital, Third Military Medical University (Chongqing, China), the Shaanxi Provincial People's Hospital, the PLA 180 Hospital and Daping Hospital, Third Military Medical University, which covered the costs of the different reagents for the serum tests.

\section{Availability of data and materials}

All data generated or analyzed during this study are included in this published article.

\section{Authors' contributions}

KQZ was responsible for all aspects of the study. SJL, JPZ and KQZ designed the study and cooperated with pathologist and clinical laboratories to analyze the results. YW collect the serum from the healthy individuals, and analyzed the STK1 and PSA of those individuals. JJY, XLZ and CMW were responsible for the collection of tissues and serum samples. HBM, EH and SS analyzed the data and cooperated in the writing of the manuscript. Beside the responsibility for the development, testing and production of the TK1 antibody, JZ also participated in the planning of the project. All authors provided final approval for publication.

\section{Ethics approval and consent to participate}

All procedures performed in studies involving human participants were in accordance with the ethical standards of the institutional and/or national research committee and with the 1964 Declaration of Helsinki and its later amendments or comparable ethical standards. (Health Management Centre of PLA 180 Hospital, Quanzhou, China, No. LL2009003). Informed consent was obtained from all individual participants included in the study.

\section{Patient consent for publication}

All patients provided consent for the use of their clinical data in this study.

\section{Competing interests}

The authors SJL, JPZ, YW, KQZ, JJY, XLZ, CMW, HBM, $\mathrm{EH}$ and SS declare no conflict of interest. JZ is the owner of Sino-Swed Tongkang Bio-Tech Inc., the company that produced the TK1 antibody.

\section{References}

1. Global Burden of Disease Cancer Collaboration; Fitzmaurice C, Dicker D, Pain A, Hamavid H, Moradi-Lakeh M, MacIntyre MF, Allen C, Hansen G, Woodbrook R, Wolfe C, et al: The global burden of cancer 2013. JAMA Oncol 1: 505-527, 2015.

2. Hoffman RM, Meisner AL, Arap W, Barry M, Shah SK, Zeliadt SB and Wiggins CL: Trends in United States prostate cancer incidence rates by age and stage, 1995-2012. Cancer Epidemiol Biomarkers Prev 25: 259-263, 2016.

3. Schröder FH, Hugosson J, Roobol MJ, Tammela TL, Zappa M, Nelen V, Kwiatkowski M, Lujan M, Määttönen L, Lilja H, et al: Screening and prostate cancer mortality: Results of the European randomised study of screening for prostate cancer (ERSPC) at 13 years of follow-up. Lancet 384: 2027-2035, 2014.

4. Siegel RL, Miller KD and Jemal A: Cancer statistics, 2017. CA Cancer J Clin 67: 7-30, 2017.

5. Siegel R, Naishadham D and Jemal A: Cancer statistics, 2013. CA Cancer J Clin 63: 11-30, 2013.

6. Chen W, Zheng R, Baade PD, Zhang S, Zeng H, Bray F, Jemal A, Yu XQ and He J: Cancer statistics in China 2015. CA Cancer J Clin 66: 115-132, 2016.

7. Gleason DF: Classification of prostatic carcinomas. Cancer Chemother Rep 50: 125-128, 1966.

8. Amin MB, Schultz DS and Zarbo RJ: Analysis of cribriform morphology in prostatic neoplasia using antibody to high-molecular-weight cytokeratins. Arch Pathol Lab Med 118: 260-264, 1994.

9. Epstein JI, Allsbrook WC Jr, Amin MB and Egevad LL: The 2005 International Society of Urological Pathology (ISUP) consensus conference on gleason grading of prostatic carcinoma. Am J Surg Pathol 29: 1228-1242, 2005.

10. Eggener SE, Badani K, Barocas DA, Barrisford GW, Cheng JS, Chin AI, Corcoran A, Epstein JI, George AK, Gupta GN, et al: Gleason 6 prostate cancer: Translating biology into population health. J Urol 194: 626-634, 2015.

11. Epstein JI, Egevad L, Amin MB, Delahunt B, Srigley JR and Humphrey PA; Grading Committee: The 2014 International Society of Urological Pathology (ISUP) consensus conference on gleason grading of prostatic carcinoma: Definition of grading patterns and proposal for a new grading system. Am J Surg Pathol 40: 244-252, 2016.

12. Gordetsky J and Epstein J: Grading of prostatic adenocarcinoma: Current state and prognostic implications. Diagn Pathol 11: 25, 2016.

13. Suzuki H, Komiya A, Kamiya N, Imamoto T, Kawamura K, Miura J, Suzuki N, Nakatsu H, Hata A and Ichikawa T: Development of a nomogram to predict probability of positive initial prostate biopsy among Japanese patients. Urology 67: 131-136, 2006.

14. Shipitsin M, Small C, Choudhury S, Giladi E, Friedlander S, Nardone J, Hussain S, Hurley AD, Ernst C, Huang YE, et al: Identification of proteomic biomarkers predicting prostate cancer aggressiveness and lethality despite biopsy-sampling error. $\mathrm{Br}$ J Cancer 111: 1201-1212, 2014.

15. Epstein JI, Feng Z, Trock BJ and Pierorazio PM: Upgrading and downgrading of prostate cancer from biopsy to radical prostatectomy: Incidence and predictive factors using the modified Gleason grading system and factoring in tertiary grades. Eur Urol 61: 1019-1024, 2012.

16. Pezaro CJ, Omlin A, Lorente D, Nava RD, Ferraldeschi R, Bianchini D, Mukherji D, Riisnaes R, Altavilla A, Crespo M, et al: Visceral disease in castration-resistant prostate cancer. Eur Urol 65: 270-273, 2014.

17. Ramakrishnan VM, Bossert K, Singer G, Lehmann K and Hefermehl LJ: The impact of the 2005 International Society of Urological Pathology Gleason grading consensus on active surveillance for prostate cancer. Cent European J Urol 70: 344-348, 2017.

18. Lima NG, Soares Dde F and Rhoden EL: Importance of prostate-specific antigen (PSA) as a predictive factor for concordance between the Gleason scores of prostate biopsies and RADICAL prostatectomy specimens. Clinics (Sao Paulo) 68: 820-824, 2013.

19. Liu X, Chen X, Rycaj K, Chao HP, Deng Q, Jeter C, Liu C, Honorio S, Li H, Davis T, et al: Systematic dissection of phenotypic, functional, and tumorigenic heterogeneity of human prostate cancer cells. Oncotarget 6: 23959-23986, 2015.

20. Sfanos KS and De Marzo AM: Prostate cancer and inflammation: The evidence. Histopathology 60: 199-215, 2012. 
21. Grimm P, Billiet I, Bostwick D, Dicker AP, Frank S, Immerzeel J, Keyes M, Kupelian P, Lee WR, Machtens S, et al: Comparative analysis of prostatespecific antigen free survival outcomes for patients with low, intermediate and high risk prostate cancer treatment by radical therapy. Results from the Prostate Cancer Results Study Group. BJU Int 109 (Suppl 1): S22-S29, 2012.

22. Thompson I, Thrasher JB, Aus G, Burnett AL, Canby-Hagino ED, Cookson MS, D'Amico AV, Dmochowski RR, Eton DT, Forman JD, et al: Guideline for the management of clinically localized prostate cancer: 2007 update. J Urol 177: 2106-2131, 2007.

23. Wilt TJ, MacDonald R, Rutks I, Shamliyan TA, Taylor BC and Kane RL: Systematic review: Comparative effectiveness and harms of treatments for clinically localized prostate cancer. Ann Int Med 148: 435-448, 2008.

24. Mitchell JM: Urologists' use of intensity-modulated radiation therapy for prostate cancer. N Engl J Med 369: 1629-1637, 2013.

25. Ilic D, Neuberger MM, Djulbegovic M and Dahm P: Screening for prostate cancer. Cochrane Database Syst Rev 31: CD004720, 2013.

26. Herschman JD, Smith DS and Catalona WJ: Effect of ejaculation on serum total and free prostate-specific antigen concentrations. Urology 50: 239-243, 1997.

27. Pron G: Prostate-specific antigen (PSA)-based population screening for prostate cancer: An evidence-based analysis. Ont Health Technol Assess Ser 15: 1-64, 2015.

28. Skog S, He E and Haghdoost S: Prevention and early detection of human tumor. LAP Lambert Academic Publishing, p74, 2017.

29. Wu JT: Circulating tumor markers of the new millennium. AACC Press, New York, NY, pp115-117, 2002.

30. Bouchardy C, Fioretta G, Rapiti E, Verkooijen HM, Rapin $\mathrm{CH}$, Schmidlin F, Miralbell R and Zanetti R: Recent trends in prostate cancer mortality show a continuous decrease in several countries. Int J Cancer 123: 421-429, 2008.
31. Zhou J, He E and Skog S: The proliferation marker thymidine kinase 1 in clinical use (Review). Mol Clin Oncol 1: 18-28, 2013.

32. Letocha H, Eklöv S, Gronowitz S, Norlen BJ and Nilsson S: Deoxythymidine in staging of prostatic carcinoma. Prostate 29: 15-19, 1996

33. Li SJ, Wu G, Ye J, Zhang X, Cheng F, Zhang KQ and Yan-Feng J: The purpose of blood serum thymidine kinase level in early screening of prostatic carcinoma. Immun J 27: 459-461, 2011

34. Jagarlamudi, KK, Hansson LO and Eriksson S: Breast and prostate cancer patients differ significantly in their serum thymidine kinase 1 (TK1) specific activities compared with those hematological malignancies and blood donors: Implications of using serum TK1 as a biomarker. BMC Cancer 15: 66, 2015.

35. Aufderklamm S, Hennenlotter J, Todenhoefer T, Gakis G, Schilling D, Vogel U, Kuehs U: Dlugosch J, Knapp J, Merseburger A, et al: XPA-210: A new proliferation marker determines locally advanced prostate cancer and is a predictor of biochemical recurrence. World J Urol 30: 547-552, 2012.

36. Na Y, Sun Z, Ye Z and Sun Y: Guidelines for the diagnosis and treatment of Urology Surgery in China. In: III Guidelines for the diagnosis and treatment of prostate cancer. Ming L, Su G and He D (eds). People's Health Press, Beijing, pp32-84, 2007.

37. Grönberg H, Adolfsson J, Aly M, Nordström T, Wiklund P, Brandberg Y, Thompson J, Wiklund F, Lindberg J, Clements M, et al: Prostate cancer screening in men aged 50-69 years (STHLM3): A prospective population-based diagnostic study. Lancet Oncol 16: 1667-1676, 2015.

38. Chen ZH, Huang SQ, Wang Y, Yang AZ, Wen J, Xu XH, Chen Y, Chen QB, Wang YH, He E, et al: Serological thymidine kinase 1 is a biomarker for early detection of tumours-a health screening study on 35,365 people using a sensitive chemiluminescent dot blot assay. Sensor (Basel) 11: 11064-11080, 2011. 